\title{
INVESTIGATION OF THE POSSIBLE MODERATORS OF ALCOHOL-RELATED INTIMATE PARTNER VIOLENCE IN SUB-SAHARAN AFRICA
}

\author{
Ekpenyong M.S. ${ }^{1}$ and Tawari E. P. ${ }^{2 *}$ \\ ${ }^{1}$ Department of Public Health, Faculty of Education, Health and wellbeing, University of \\ Wolverhampton, United Kingdom. \\ ${ }^{2}$ Department of Chemical Pathology, Faculty of Basic Medical Sciences, College of Health \\ Science, Niger Delta University, Bayelsa State, Nigeria. \\ *Corresponding author: +234(0)7035573306; perebi8@yahoo.com
}

\section{Cite this article:}

Ekpenyong M.S., Tawari E. P. (2021), Investigation of the Possible Moderators of Alcohol-Related Intimate Partner Violence in SubSaharan Africa. African Journal of Biology and Medical Research 4(2), 67-83. DOI: $10.52589 / A J B M R-$ OAEBS2XL.

\section{Manuscript History}

Received: 26 April 2021

Accepted: 17 May 2021

Published: 21 May 2021

Copyright $\odot 2020$ The Author(s). This is an Open Access article distributed under the terms of Creative Commons AttributionNonCommercial-NoDerivatives 4.0 International (CC BY-NC-ND 4.0 ), which permits anyone to share, use, reproduce and redistribute in any medium, provided the original author and source are credited.
ABSTRACT: Alcohol-related intimate partner violence (IPV) is a serious public health issue which has attracted a lot of research and debates. While some studies have reported the relationship between alcohol and IPV to be linear, others have reported threshold effects. While some studies have found the link to be strong, others have reported it to be weak or to show no association. The aim of this study was to determine the possible moderators on the alcohol-IPV link in sub-Saharan Africa. For the quantitative study, secondary analysis and meta-analysis were used to analyze cross-sectional data from the demographic and health surveys of ten countries in sub-Saharan Africa (Burkina Faso, Ghana, Kenya, Liberia, Malawi, Nigeria, Sao Tome and Principe, Tanzania, Zambia, and Zimbabwe). Logistic regression analysis of possible moderators of the alcohol-IPV link was determined in ten sub-Saharan African countries. The nature of moderation was different among countries. The results of this study can be applied in planning country-specific and multi-faceted intervention programmes.

KEYWORDS: Alcohol, Violence, Sub-Saharan Africa. 


\section{INTRODUCTION}

Intimate partner violence is defined as any physical, psychological or sexual harm that is caused by the actions of a presently or previously intimate partner (WHO, 2012). Prevalent rates are different across countries with rates between 11-52\% in developing countries (Gage, 2005; Kishor \& Johnson, 2004). The health and social consequences of intimate partner violence are tremendous. IPV has been reported to lead to physical injuries, loss of pregnancy and complications during pregnancy (WHO, 2010). It can also result in emotional problems such as depression and suicide (WHO, 2010) and victims have been reported to resort to the use of drugs and alcohol as a means of coping with abuse (Krug et al., 2002). Injuries from IPV may sometimes be fatal. For example, one study in the US estimated that of the homicides recorded in a period of 26 years, 11 percent were committed by intimate partners (WHO, 2010). Problems also affect family, friends and future relationships (WHO, 2010). In addition, children who witness parental violence are at an increased risk of alcohol abuse (Trocki \& Caetano, 2003) and perpetrating violence later in life.

Risk factors such as young age, low education, occupation, experiencing parental violence, drug and alcohol use, controlling behavior by the husband (Stith et al., 2004) and justification of wife beating (Uthman et al., 2010) have been associated with an increase in the odds of IPV; of these, alcohol consumption has been steadily implicated (Taft et al., 2010; WHO, 2010). Explanations of the role of alcohol on IPV range from ascribing a causal role to seeing alcohol as an excuse for violence. While some have posited that this link is as a result of the direct pharmacological effect of alcohol on cognitive behavior, others have argued that the relationship is as a result of the presence of confounders or simply that alcohol is just an excuse for violence (Foran \& O'Leary, 2008). Alcohol has also been reported to have a linear effect by some studies and a threshold effect by other studies. For example, Bangdiwala et al. (2011) observed a statistically significant increase in the risk of experiencing IPV by women in low and middle income countries when their husbands consume alcohol, with the risk increasing with more alcohol intake. In contrast, others argue that alcohol abuse rather than alcohol consumption is a contributory factor to IPV (Tumwesigye et al., 2012; Gage, 2005). Furthermore, there is reluctance among feminist groups to attribute a causal relationship between alcohol and IPV because of the belief that it will remove responsibility from perpetrators (Foran \& O'Leary, 2008). In addition to the above controversy, there are debates on the strength of the association between alcohol and IPV.

Recent researches of the alcohol-IPV link have called for more research on the moderators of this link (Foran et al., 2012; Foran \& O'leary, 2008) especially in Africa (Tumwesigye et al., 2012). The dearth of literature on alcohol-related IPV in sub-Saharan Africa and the limited research on possible moderators motivated this research. Knowledge and understanding of alcohol-related IPV across diverse cultures using methods that can be compared will aid development of policies suitable for the societies in which they are applied. An understanding of the moderators of the alcohol-IPV link will assist while planning interventions on alcoholrelated IPV by tackling not just alcohol but including intervention programs that will also tackle the contributing factors.

The aim of this study was to examine the possible moderators on the alcohol-IPV link in subSaharan Africa. 


\section{METHODOLOGY}

For the quantitative study, secondary analysis and meta-analysis were used to analyze crosssectional data from the demographic and health surveys of ten countries in sub-Saharan Africa (Burkina Faso, Ghana, Kenya, Liberia, Malawi, Nigeria, Sao Tome and Principe, Tanzania, Zambia, and Zimbabwe). Since the aim of this research was to determine the relationship between alcohol and IPV in sub-Saharan Africa, a quantitative research design was adopted because it is an appropriate method for showing associations and quantifying relationships between variables (Welman et al., 2005).

In order to study the relationship and moderators of the alcohol-IPV link in sub-Saharan Africa, a secondary analysis of previously conducted primary studies of ten countries in sub-Saharan Africa was carried out. This was the method of choice for this research as it took a cross national perspective which required that data from several countries in sub-Saharan Africa be analyzed. This study also aimed to determine the association between alcohol and IPV; a larger sample size was used. Data sets for this study were also easier to access and this raised little or no ethical issues as respondents were already made anonymous.

\section{Data Collection Methods}

This study did not collect primary data but accessed data of the demographic and health survey of ten sub-Saharan Africa countries (Burkina Faso, Ghana, Kenya, Liberia, Malawi, Nigeria, Sao Tome and Principe, Tanzania, Zambia, and Zimbabwe). Large sample sizes were used with high response rate, thereby ensuring that statistically significant relationships are detected. Access to the data sets was gained by requesting permission from Demographic and Health Survey (DHS); approval from DHS was granted by email. Data was identified using the domestic violence questionnaire. The identified data sets were downloaded to the researcher's personal computer using SPSS 19 software. Variables in the study were identified using the DHS recode manual.

\section{Sample/Sampling Strategy}

This study used quota sampling to identify DHS surveys conducted between 2006 and 2011, made available by 2012 in sub-Saharan Africa. For this research, only countries from subSaharan Africa were included because the main independent variable (alcohol consumption) was not measured in North African countries as consumption is prohibited. The data for each country was the most recent. This was to ensure that results reflect the current strength of the alcohol-IPV link and that recommendations are made based on current evidence. All datasets included questions on domestic violence and covered the topic of alcohol consumption and frequency at which husbands/partners get drunk because of the fact that the focus of this study is on alcohol-related IPV.

\section{Reliability and Validity}

Reliability and validity were ensured by strategies and methods that have been used to study the alcohol-IPV link in several literatures (Foran \& O'Leary, 2008; Tumwesigye et al., 2012). 


\section{DATA ANALYSIS}

Data analysis was carried out using SPSS 19 and Revman Meta-analysis software.

Hierarchical Logistic Regression: Using the method described by Field (2005), hierarchical logistic regression was used to study the moderator effects of the independent variables on the alcohol-IPV link. In order to account for the complex sampling used in the DHS survey, the probability of being administered the domestic violence questionnaire and to adjust for the probability of non-response (DHS, 2012), all analyses were conducted using the domestic violence sample weight.

\section{Ethical Issues}

One of the ethical issues with conducting a secondary data analysis is the permission to access datasets. Approval was sought by the researcher and access was granted. The DHS also operated a no data sharing policy. To ensure data protection, memory sticks and computers were password protected to guard against unauthorized access. It is also important to confirm that the data obtained from the primary study was ethically obtained. The primary DHS study was done with the informed consent of the subjects and confidentiality obtained with the National ethics committees of the different countries approving the surveys. Furthermore, the datasets were made anonymous by removing all identifier information. For this study, ethical approval was sought by the researcher and approval was granted by the ethics committee of the University of Wolverhampton.

\section{RESULTS}

\section{Moderators of the Alcohol-Intimate Partner Violence Link}

In order to investigate possible moderators of the alcohol-IPV link, multivariate logistic regression was carried out. Moderators were grouped based on the socio-ecological framework into individual, relationship and societal factors, giving rise to three models. Each of the models was built with alcohol and the moderators in the first block and a product of alcohol and possible moderators in the second block.

\section{Individual Level Moderators}

Three individual level factors were investigated for moderator effect on the alcohol-IPV link. These include employment status of the man, occupation and wealth index (as a proxy for income). The result of this model is presented in Table 1 ( $a, b$ and $c$ ). The full model consists of four variables that are independent and five interaction terms. Though wealth index was not a significant contributor to the main effect in Burkina Faso, the interaction term with alcohol abuse was statistically significant: $\mathrm{OR}=0.50$ (95\% CI, 0.32-0.98) in middle index and $\mathrm{OR}=0.35$ (95\% CI, 0.21-0.59, $\mathrm{p}<0.05)$ indicating that being rich decreases the odds of alcohol-related IPV in Burkina Faso. Of the three individual factors investigated for moderator effect, only wealth index moderated the alcohol-IPV link in Kenya: OR=0.53 (95\% CI, 0.28-0.99), Tanzania: OR=0.41 (95\% CI, 0.20-0.84) and Liberia: OR=2.04 (95\% CI, 1.163.59) with moderation reaching statistical significance in the rich index group. This result shows that being rich reduces the odds of alcohol-related violence in Kenya and Tanzania while it increases the likelihood of alcohol-related IPV in Liberia. Occupation and employment status did not moderate the alcohol-IPV link in any of the ten countries in this study. The full model was able to classify about $58.3 \%$ to $83.3 \%$ of the cases. 


\section{Table 1a: Effect of Individual Level Moderators on the Alcohol-Ipv Link in Sub-Saharan Africa}

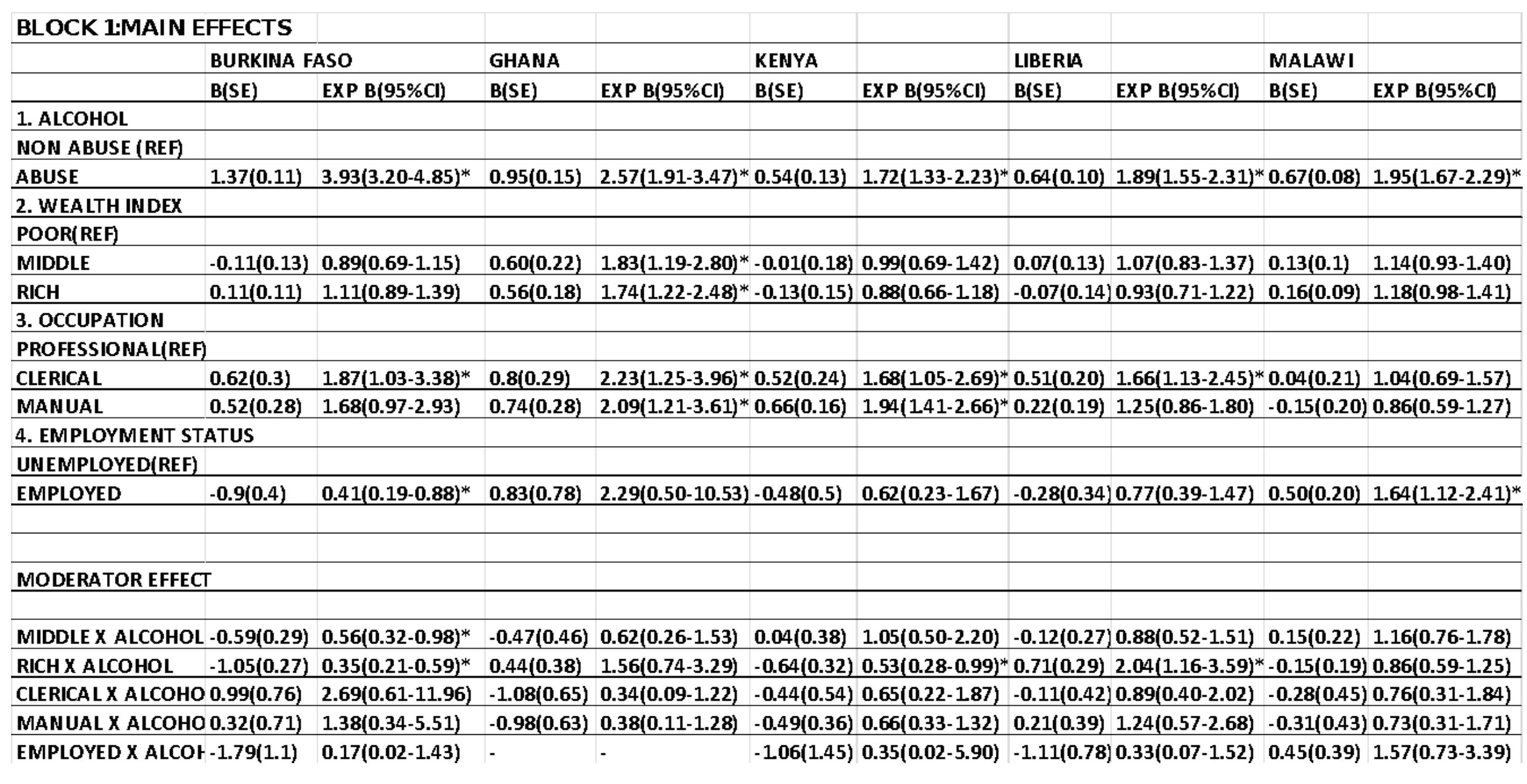

$* \mathrm{p}<0.05$ 


\section{Table 1b: Effect of Individual Level Moderators on the Alcohol-Ipv Link in Sub-Saharan Africa}

\begin{tabular}{|c|c|c|c|c|c|c|c|c|c|c|}
\hline \multicolumn{2}{|c|}{ BLOCK 1:MAIN EFFECTS } & \multirow[b]{3}{*}{ EXP B $(95 \% \mathrm{Cl})$} & & & & & \multirow{3}{*}{$\begin{array}{l}\text { ZAMBIA } \\
\text { B(SE) } \\
\end{array}$} & \multirow[b]{3}{*}{ EXP B $(95 \% \mathrm{Cl})$} & & \\
\hline & NIGERIA & & \multicolumn{2}{|c|}{ SAO TOME AND PRINCIPE } & \multicolumn{2}{|l|}{ TANZANIA } & & & \multicolumn{2}{|l|}{ ZIMBABWE } \\
\hline & $\mathrm{B}(\mathrm{SE})$ & & $\mathrm{B}(\mathrm{SE})$ & EXP B $(95 \% \mathrm{Cl})$ & $\mathrm{B}(\mathrm{SE})$ & EXP B $(95 \% \mathrm{Cl})$ & & & $\mathrm{B}(\mathrm{SE})$ & EXP B $(95 \% \mathrm{Cl})$ \\
\hline \multicolumn{11}{|l|}{ 1. ALCOHOL } \\
\hline \multicolumn{11}{|l|}{ NON ABUSE (REF) } \\
\hline ABUSE & $1.26(0.09)$ & $3.53(2.98-4.18)^{*}$ & $1.66(0.22)$ & $5.25(3.39-8.13)^{*}$ & $1.09(0.16)$ & $2.98(2.20-4.03)^{*}$ & $0.66(0.09)$ & $1.93(1.63-2.28)^{*}$ & $0.68(0.1)$ & $1.97(1.63-2.89)^{*}$ \\
\hline \multicolumn{11}{|l|}{ 2. WEALTH INDEX } \\
\hline \multicolumn{11}{|l|}{ POOR(REF) } \\
\hline MIDDLE & $0.24(0.08)$ & $1.28(1.08-1.50)^{*}$ & $-0.03(0.3)$ & $0.97(0.54-1.76)$ & $0.02(0.20)$ & $1.02(0.69-1.51)$ & $0.26(0.12)$ & $1.29(1.03-1.62)^{*}$ & $-0.23(0.14)$ & $0.79(0.61-1.03)$ \\
\hline RICH & $-0.07(0.08)$ & $0.94(0.8-1.09)$ & $0.07(0.23)$ & $1.07(0.68-1.68)$ & $0.30(0.17)$ & $1.35(0.98-1.87)$ & $0.44(0.11)$ & $1.55(1.25-1.93)^{*}$ & $-0.49(0.11)$ & $0.61(0.49-0.76)^{*}$ \\
\hline \multicolumn{11}{|l|}{ 3. OCCUPATION } \\
\hline \multicolumn{11}{|l|}{ PROFESSIONAL(REF) } \\
\hline CLERICAL & $-0.09(0.12)$ & $0.92(0.73-1.16)$ & $-0.60(0.37)$ & $0.55(0.27-1.13)$ & $0.95(0.50)$ & $2.59(0.98-6.82)$ & $0.61(0.19)$ & $1.83(1.27-2.64)^{*}$ & $0.24(0.23)$ & $1.27(0.81-2.00)$ \\
\hline MANUAL & $-0.09(0.12)$ & $0.92(0.73-1.15)$ & $-0.43(0.40)$ & $0.65(0.30-1.42)$ & $1.70(0.40)$ & 5.45(2.48-11.97) & $0.67(0.18)$ & $1.95(1.38-2.77)^{*}$ & $0.34(0.20)$ & $1.41(0.95-2.10)$ \\
\hline \multicolumn{11}{|c|}{ 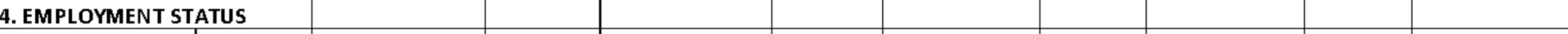 } \\
\hline \multicolumn{11}{|c|}{ UNEMPLOYED(REF) } \\
\hline EMPLOYED & $-0.06(0.4)$ & $0.94(0.43-2.07)$ & - & - & $1.35(1.45)$ & $3.84(0.22-66.00)$ & $0.50(0.26)$ & $1.66(0.98-2.79)$ & $0.40(0.17)$ & $1.49(1.08-2.06)^{*}$ \\
\hline \multicolumn{11}{|l|}{ MODERATOR EFFECT } \\
\hline MIDDLE X ALCOHOL & $-0.23(0.25)$ & $0.80(0.49-1.29)$ & $0.4(0.73)$ & $1.49(0.36-6.20)$ & $-0.67(0.43)$ & $0.51(0.22-1.19)$ & $0.23(0.24)$ & $1.25(0.79-1.99)$ & $-0.25(0.27)$ & $0.78(0.46-1.33)$ \\
\hline RICH X ALCOHOL & $-0.39(0.22)$ & $0.68(0.45-1.04)$ & $-1.08(0.53)$ & $0.34(0.12-0.96)^{*}$ & $-0.88(0.36)$ & $0.41(0.20-0.84)^{*}$ & $0.28(0.22)$ & $1.32(0.85-2.05)$ & $-0.07(0.23)$ & $0.93(0.59-1.46)$ \\
\hline CLERICAL X ALCOHO & $0.07(0.36)$ & $1.07(0.53-2.15)$ & $-1.05(1.13)$ & $0.35(0.04-3.21)$ & $0.16(0.99)$ & $1.18(0.17-8.14)$ & $0.02(0.37)$ & $1.03(0.49-2.13)$ & $0.03(0.48)$ & $1.03(0.40-2.64)$ \\
\hline MANUAL X ALCOHO & $0.40(0.35)$ & $1.50(0.76-2.96)$ & $-2.07(1.17)$ & $0.13(0.01-1.26)$ & $0.23(0.80)$ & $1.26(0.26-5.96)$ & $0.07(0.36)$ & $1.07(0.53-2.16)$ & $-0.01(0.43)$ & $1.00(0.43-2.31)$ \\
\hline $\begin{array}{l}\text { EMPLOYED X ALCOH } \\
* p<0.05\end{array}$ & $-3.55(1.89)$ & $0.03(0.001-1.17)$ & $\mid-$ & |- & $\mid-$ & $\mid$ & $|0.45(0.53)|$ & $|1.58(0.56-4.44)|$ & $|0.30(0.33)|$ & $|1.35(0.71-2.57)|$ \\
\hline
\end{tabular}


Table 1c: Model Summary

\begin{tabular}{|c|c|c|c|c|}
\hline COUNTRIES & Chi Square & Cox \&Snell $R^{2}$ & Nigelkerke R² & \%Cases correctly classified \\
\hline BURKINA FASO & $X^{2}(11, N=3617)=186.12, p=0.000$ & 0.053 & 0.09 & $83.30 \%$ \\
\hline GHANA & $X^{2}(11, N=1019)=67.94, p=0.000$ & 0.075 & 0.103 & $66.40 \%$ \\
\hline KENYA & $X^{2}(11, N=1251)=52.70, p=0.000$ & 0.047 & 0.063 & $61.80 \%$ \\
\hline LIBERIA & $X^{2}(11, N=2072)=59.81, p=0.000$ & 0.033 & 0.044 & $58.40 \%$ \\
\hline MALAWI & $X^{2}(11, N=3254)=85.94, p=0.000$ & 0.028 & 0.039 & $64.70 \%$ \\
\hline NIGERIA & $X^{2}(11, N=6424)=246.58, p=0.000$ & 0.044 & 0.064 & $72.70 \%$ \\
\hline SAO TOME\& PRINCIPE & $X^{2}(11, N=560)=77.24, p=0.000$ & 0.142 & 0.193 & $70.90 \%$ \\
\hline TANZANIA & $X^{2}(10, N=972)=81.95, p=0.000$ & 0.09 & 0.121 & $64.60 \%$ \\
\hline ZAMBIA & $X^{2}(11, N=2614)=95.78, p=0.000$ & 0.04 & 0.054 & $58.30 \%$ \\
\hline ZIMBABWE & $X^{2}(11, N=1972)=90.72, p=0.000$ & 0.048 & 0.065 & $60.60 \%$ \\
\hline
\end{tabular}

\section{Relationship Factors}

Length of marriage, jealousy and marital status were three of the relationship factors investigated for moderator effects in this study. The full model was constructed by entering alcohol and the three relationship variables in the first block and the interaction terms in the second block. The model was made up of four independent variables and five interaction terms. In Burkina Faso, even though the main effect of length of marriage was not statistically significant, the interaction term with alcohol was significant with the interaction term reducing the strength of the alcohol-IPV link. In contrast, being in marriage for a long time increased the strength of the alcohol-IPV link in Nigeria: OR=1.62 $(1.08-2.42, \mathrm{p}<0.05)$ and Zambia: $\mathrm{OR}=4.10(1.86-9.00, \mathrm{p}<0.05)$, with the moderator effect reaching statistical significance in men who have been married for 10-19 years and over 30 years respectively. Even though the main effect of jealousy was significant in all the countries, the moderator effect showed statistical significance only in Tanzania with jealousy increasing the strength of the alcoholIPV link. Surprisingly, the interaction term between alcohol and marital status shows that the strength of the alcohol-IPV link is weakened in couples who are cohabiting in Kenya. None of the relationship factors emerged as a significant moderator of the alcohol-IPV link in Ghana, Liberia, Malawi, Sao Tome and Principe, and Zimbabwe. The results of the effect of relationship moderators on the alcohol-IPV link are presented in Table 2 ( $a, b$ and c) below: 


\section{Table 2a: Effect Of Relationship Moderators On The Alcohol-Ipv Link In Ssa}

\begin{tabular}{|c|c|c|c|c|c|c|c|c|c|c|}
\hline \multicolumn{11}{|l|}{ BLOCK 1:MAIN EFFECTS } \\
\hline & BURKINA FASO & & GHANA & & KENYA & & LIBERIA & & MALAWI & \\
\hline & $\mathrm{B}(\mathrm{SE})$ & EXP B $(95 \% \mathrm{Cl})$ & B(SE) & $\operatorname{EXP} B(95 \% \mathrm{Cl})$ & $\mathrm{B}(\mathrm{SE})$ & EXP B $(95 \% \mathrm{Cl})$ & $B(S E)$ & EXP B $(95 \% \mathrm{Cl})$ & $B(S E)$ & EXP B $(95 \% \mathrm{Cl})$ \\
\hline \multicolumn{11}{|l|}{ 1. ALCOHOL } \\
\hline \multicolumn{11}{|l|}{ NON ABUSE (REF) } \\
\hline ABUSE & $1.39(0.11)$ & $4.00(3.26-4.93)^{*}$ & $0.78(0.16)$ & $2.19(1.61-2.97)^{*}$ & $0.44(0.14)$ & $1.56(1.19-2.04)^{*}$ & $0.72(0.10)$ & $2.06(1.68-2.53)^{*}$ & $0.56(0.09)$ & $1.75(1.48-2.07)^{*}$ \\
\hline \multicolumn{11}{|l|}{ 2. LENGTH OF MARRIAGE } \\
\hline \multicolumn{11}{|l|}{ 0-gYEARS (REF) } \\
\hline 10-19YEARS & $0.001(0.1)$ & $1.00(0.82-1.23)$ & $0.38(0.18)$ & $1.46(1.03-2.08)^{*}$ & $0.29(0.15)$ & $1.33(0.99-1.78)$ & $-0.12(0.11)$ & $0.88(0.71-1.10)$ & $0.17(0.09)$ & $1.18(0.98-1.42)$ \\
\hline 20-29YEARS & $0.08(0.13)$ & $1.08(0.83-1.41)$ & $0.15(0.2)$ & $1.16(0.78-1.72)$ & $0.47(0.18)$ & $1.6(1.12-2.28)^{*}$ & $-0.73(0.14)$ & $0.48(0.37-0.64)^{*}$ & $-0.08(0.12)$ & $0.93(0.74-1.16)$ \\
\hline 30+YEARS & - & - & $0.46(0.32)$ & $1.59(0.85-2.97)$ & $2.71(0.57)$ & $15.06(4.89-46.34)^{*}$ & $-0.94(0.54)$ & $0.39(0.14-1.12)$ & $-0.28(0.24)$ & $0.76(0.48-1.21)$ \\
\hline \multicolumn{11}{|l|}{ 3. JEALOUSY } \\
\hline \multicolumn{11}{|l|}{ NO(REF) } \\
\hline YES & $0.45(0.1)$ & $1.57(1.30-1.89)^{*}$ & $1.16(0.15)$ & $3.19(2.37-4.30)^{*}$ & $1.05(0.13)$ & $2.86(2.21-3.71)^{*}$ & $1.19(0.11)$ & $3.30(2.64-4.12)^{*}$ & $1.60(0.08)$ & $4.95(4.21-5.82)^{*}$ \\
\hline \multicolumn{11}{|l|}{ 4. MARTIAL STATUS } \\
\hline \multicolumn{11}{|l|}{ MARRIED(REF) } \\
\hline COHABTING & $0.26(0.18)$ & $1.29(0.91-1.84)$ & $0.49(0.27)$ & $1.64(0.97-2.77)^{\wedge}$ & $0.74(0.33)$ & $2.10(1.10-4.00)^{*}$ & $-0.09(0.10)$ & $0.92(0.75-1.12)$ & $-0.27(0.12)$ & $0.76(0.61-0.96)^{*}$ \\
\hline \multicolumn{11}{|l|}{ MODERATOR EFFECT } \\
\hline 10-19YEARS X ALCOHOL & $-0.83(0.24)$ & $0.44(0.28-0.69)^{*}$ & $-0.07(0.38)$ & $0.94(0.44-1.97)$ & $-0.45(0.32)$ & $0.64(0.35-1.19)$ & $0.23(0.24)$ & $1.25(0.79-1.99)$ & $0.17(0.20)$ & $1.19(0.81-1.74)$ \\
\hline 20-29YEARSX ALCOHOL & $-0.57(0.30)$ & $0.57(0.31-1.02)$ & $0.05(0.42)$ & $1.05(0.47-2.37)$ & $-0.22(0.37)$ & $0.80(0.39-1.67)$ & $0.48(0.29)$ & $1.62(0.91-2.87)$ & $-0.12(0.24)$ & $0.89(0.56-1.42)$ \\
\hline 30+YEARS X ALCOHOL & - & - & $0.56(0.67)$ & $1.74(0.47-6.46)$ & $0.49(1.44)$ & $1.64(0.10-27.73)$ & $1.60(1.17)$ & $4.95(0.50-49.30)$ & $-0.24(0.47)$ & $0.78(0.31-1.99)$ \\
\hline JEALOUSY X ALCOHOL & $-0.13(0.21)$ & $0.88(0.58-1.36)$ & $-0.40(0.32)$ & $0.67(0.36-1.24)$ & $-0.51(0.28)$ & $0.60(0.35-1.04)$ & $-0.06(0.23)$ & $0.94(0.60-1.49)$ & $-0.01(0.17)$ & $1.01(0.72-1.41)$ \\
\hline $\begin{array}{l}\text { COHABTINGX ALCOHOL } \\
*_{\mathrm{p}}<0.05\end{array}$ & $-0.26(0.38)$ & $0.77(0.36-1.62)$ & $-0.47(0.57)$ & $0.63(0.20-1.92)$ & $|-1.41(0.71)|$ & $0.25(0.06-0.98)^{*}$ & $0.18(0.21)$ & $1.20(0.79-1.82)$ & $0.36(0.24)$ & $1.43(0.90-2.27)$ \\
\hline
\end{tabular}


Table 2b: Effect Of Relationship Moderators On The Alcohol-Ipv Link In Ssa

\begin{tabular}{|c|c|c|c|c|c|c|c|c|c|c|}
\hline \multicolumn{11}{|l|}{ BLOCK 1:MAIN EFFECTS } \\
\hline & NIGERIA & & \multicolumn{2}{|c|}{ SAO TOME AND PRINCIPE } & TANZANIA & & ZAMBIA & & ZIMBABWE & \\
\hline & $\mathrm{B}(\mathrm{SE})$ & EXР B $(95 \% \mathrm{Cl})$ & $\mathrm{B}(\mathrm{SE})$ & EXP B( $(95 \% \mathrm{Cl})$ & $\mathrm{B}(\mathrm{SE})$ & EXP B(95\%Cl) & $\mathrm{B}(\mathrm{SE})$ & $\operatorname{EXP~B~}(95 \% \mathrm{Cl})$ & $\mathrm{B}(\mathrm{SE})$ & EXP B $(95 \% \mathrm{Cl})$ \\
\hline \multicolumn{11}{|l|}{ 1. ALCOHOL } \\
\hline \multicolumn{11}{|l|}{ NON ABUSE (REF) } \\
\hline ABUSE & $1.26(0.09)$ & $3.52(2.97-4.17)$ & $1.44(0.20)$ & $4.22(2.85-6.24)^{*}$ & $1.01(0.16)$ & $2.74(2.01-3.74)^{*}$ & $0.6(0.09)$ & $1.82(1.54-2.16)^{*}$ & $0.62(0.09)$ & $1.85(1.55-2.21)^{*}$ \\
\hline \multicolumn{11}{|l|}{ 2. LENGTH OF MARRIAGE } \\
\hline \multicolumn{11}{|l|}{ 0-gYEA RS (REF) } \\
\hline 10-19YEARS & $0.32(0.07)$ & $1.38(1.19-1.59)^{*}$ & $0.28(0.22)$ & $1.33(0.86-2.04)$ & $0.26(0.16)$ & $1.30(0.95-1.78)$ & $0.05(0.10)$ & $1.05(0.87-1.28)$ & $-0.02(0.10)$ & $0.99(0.81-1.20)$ \\
\hline 20-29YEARS & $0.28(0.08)$ & $1.33(1.13-1.57)^{*}$ & $0.36(0.24)$ & $1.44(0.89-2.32)$ & $0.18(0.22)$ & $1.19(0.78-1.82)$ & $0.13(0.12)$ & $1.14(0.90-1.45)$ & $-0.17(0.14)$ & $0.84(0.65-1.10)$ \\
\hline 30+YEARS & $0.16(0.13)$ & $1.17(0.91-1.51)$ & $-0.21(0.39)$ & $0.81(0.38-1.74)$ & $1.39(1.20)$ & $4.00(0.38-42.04)$ & $0.01(0.18)$ & $1.01(0.71-1.45)$ & $-0.37(0.28)$ & $0.69(0.40-1.19)$ \\
\hline \multicolumn{11}{|l|}{ 3. JEALOUSY } \\
\hline \multicolumn{11}{|l|}{ NO(REF) } \\
\hline YES & $0.77(0.06)$ & $2.15(1.90-2.43)$ & $1.56(0.20)$ & $4.77(3.24-7.01)^{*}$ & $1.06(0.16)$ & $2.90(2.14-3.93)^{*}$ & $1.07(0.09)$ & $2.91(2.44-3.46)^{*}$ & $1.08(0.09)$ & $2.95(2.48-3.53)^{*}$ \\
\hline \multicolumn{11}{|l|}{ 4. MARTIAL STATUS } \\
\hline \multicolumn{11}{|l|}{ MARRIED(REF) } \\
\hline COHABTIING & $0.23(0.18)$ & $1.25(0.89-1.77)$ & $-0.17(0.33)$ & $0.85(0.45-1.60)$ & $-0.43(0.30)$ & $0.65(0.36-1.16)$ & $0.10(0.52)$ & $1.11(0.40-3.07)$ & $-0.46(0.44)$ & $0.63(0.27-1.49)$ \\
\hline & & & & & & & & & & \\
\hline \multicolumn{11}{|l|}{ MODERATOR EFFECT } \\
\hline 10-19YEARS X ALCOHOL & $0.48(0.21)$ & $1.62(1.08-2.42)^{*}$ & $0.02(0.46)$ & $1.02(0.41-2.51\}$ & $-0.18(0.36)$ & $0.84(0.42-1.69)$ & $0.03(0.2)$ & $1.03(0.70-1.52)$ & $0.08(0.20)$ & $1.09(0.73-1.62)$ \\
\hline 20-29YEARSX ALCOHOL & $0.02(0.24)$ & $1.02(0.63-1.63)$ & $0.68(0.57)$ & $1.97(0.65-6.04)$ & $0.14(0.45)$ & $1.15(0.48-2.79)$ & $0.32(0.25)$ & $1.38(0.85-2.23)$ & $0.39(0.28)$ & $1.48(0.86-2.54)$ \\
\hline 30+YEARS X ALCOHOL & $0.56(0.40)$ & $1.75(0.79-3.87)$ & $1.10(0.83)$ & $2.99(0.58-15.33)$ & - & - & $1.41(0.40)$ & $4.10(1.86-9.00)^{*}$ & $0.87(0.64)$ & $2.39(0.69-8.31)$ \\
\hline JEALOUSY X ALCOHOL & $0.18(0.18)$ & $1.19(0.84-1.69)$ & $-0.37(0.43)$ & $0.69(0.30-1.59)$ & $0.76(0.33)$ & $2.13(1.12-4.10)^{*}$ & $-0.02(0.18)$ & $0.98(0.69-1.39)$ & $0.13(0.18)$ & $1.14(0.80-1.63)$ \\
\hline $\begin{array}{l}\text { COHABTINGX ALCOHOL } \\
*_{p}<0.05\end{array}$ & $0.65(0.45)$ & $\mid 1.92(0.80-4.58)$ & $0.48(0.79)$ & $|1.61(0.34-7.58)|$ & $-0.21(0.61)$ & $0.81(0.25-2.66)$ & $1.05(1.28)$ & $2.85(0.23-35.2)$ & $|-1.03(0.87)|$ & $0.36(0.07-1.95)$ \\
\hline
\end{tabular}


Table 2c: Model Summary

\begin{tabular}{l|l|r|r|r}
\hline & Chi Square & Cox \&Snell $R^{2}$ & Nigelkerke $R^{2} \%$ Cases correctly classified \\
\hline BURKINA FASO & $X^{2}(9, N=3707)=197.2, p=0.000$ & 0.055 & 0.093 & $83.30 \%$ \\
\hline GHANA & $X^{2}(11, N=1019)=108.99, p=0.000$ & 0.116 & 0.159 & $67.20 \%$ \\
\hline KENYA & $X^{2}(11, N=1251)=136.79, p=0.000$ & 0.119 & 0.161 & $64.80 \%$ \\
\hline LIBERIA & $X^{2}(11, N=2179)=200.77, p=0.000$ & 0.1 & 0.137 & $64.30 \%$ \\
\hline MALAWI & $X^{2}(11, N=3349)=485.48, p=0.000$ & 0.148 & 0.203 & $71.20 \%$ \\
\hline NIGERIA & $X^{2}(11, N=6436)=399.44, p=0.000$ & 0.071 & 0.101 & $73.00 \%$ \\
\hline SAO TOME\& PRINCIPE & $X^{2}(11, N=791)=153.51, p=0.000$ & 0.207 & 0.285 & $72.20 \%$ \\
\hline TANZANIA & $X^{2}(11, N=974)=105.06, p=0.000$ & 0.114 & 0.153 & $65.60 \%$ \\
\hline ZAMBIA & $X^{2}(11, N=2661)=232.82, p=0.000$ & 0.093 & 0.124 & $63.50 \%$ \\
\hline ZIMBABWE & $X^{2}(11, N=2457)=224.26, p=0.000$ & 0.095 & 0.128 & $64.00 \%$ \\
\hline
\end{tabular}

\section{Societal Level Moderators}

Three societal level variables-place of residence, male justification of wife beating and society justification of wife beating - were investigated for possible moderator effect on the alcohol-IPV link. The model was built by entering alcohol abuse and the three societal level variables into the first block, and the interaction terms of alcohol and each of the societal level variables in the second block. While the interaction between alcohol and living in a rural area reduced the odds of IPV in Malawi: $\mathrm{OR}=0.46$ (95\% CI=0.31-0.72, $\mathrm{p}<0.05)$, Zambia: $\mathrm{OR}=0.69$ (95\% CI $=0.48-0.99, \mathrm{p}<0.05)$ and Sao Tome and Principe: OR=0.46 (95\% CI=0.22-0.99, $\mathrm{p}<0.05)$, the interaction between alcohol and living in a rural area increased the odds of IPV in Nigeria: $\mathrm{OR}=1.96$ (95\% CI=1.36-2.82). While the interaction between alcohol and society's justification of wife beating increased the odds of IPV in Burkina Faso: OR=2.26 (95\% $\mathrm{CI}=1.33-3.86, \mathrm{p}<0.05)$, surprisingly the same interaction term reduced the odds of IPV in Zambia: OR=0.63 (95\% CI=0.40-0.99, p<0.05) and Ghana: $\mathrm{OR}=0.35$ (95\% CI=0.17-0.70, $\mathrm{p}<0.05)$. None of the societal level factors investigated moderated the relationship between alcohol and IPV in Liberia, Kenya, Tanzania and Zimbabwe. The entire models containing all four predictors and three interaction terms were statistically significant in all ten countries, with the models correctly classifying $57.9 \%$ to $83.4 \%$ of the cases in these countries. The results of the full models are presented in Tables 3 ( $a, b$ and $c)$ : 


\section{Table 3a: Effects Of Society Level Factors On The Alcohol-Ipv Link In Ssa}

\begin{tabular}{|c|c|c|c|c|c|c|c|c|c|c|}
\hline \multicolumn{11}{|l|}{ BLOCK 1:MAIN EFFECTS } \\
\hline & BURKINA FASO & & GHANA & & KENYA & & LIBERIA & & MALAWI & \\
\hline & $B(S E)$ & EXP B $\{95 \% a)$ & BiSE) & EXP B $(95 \%$ C $)$ & $B(S E)$ & EXP B $\{95 \%$ C $)\}$ & B(SE) & EXP B $(95 \% a)$ & $B(S E)$ & EXP B $\{95 \%$ C $)\}$ \\
\hline \multicolumn{11}{|l|}{ 1. ALCOHOL } \\
\hline \multicolumn{11}{|l|}{ NON ABUSE (REF) } \\
\hline ABUSE & $1.37(0.11)$ & $3.93(3.20-4.83)^{*}$ & $1.01(0.15)$ & $2.74(2.02 \cdot 3.70)^{*}$ & $0.53(0.13)$ & $1.69(1.31 \cdot 2.19\}^{*}$ & $0.68(0.10)$ & $1.97(1,62 \cdot 2,39)^{*}$ & $* 0.65(0.08)$ & $1.92(1.64 \cdot 2.24)^{*}$ \\
\hline \multicolumn{11}{|l|}{ 2. PLACE OF RESIDENCE } \\
\hline \multicolumn{11}{|l|}{ URBAN\{REF\} } \\
\hline RURAL & $-0.27|0.12|$ & $0.76[0.60 \cdot 0.96)^{*}$ & $-0.53(0.15)$ & $0.59(0.43-0.79)^{*}$ & $0.35(0,15)$ & $1.42(1.06-1.89)^{*}$ & $-0.22(0.10)$ & $0.81[0,66-0.98)^{*}$ & $*-0.27(0.10\}$ & $0.76(0,62 \cdot 0.93)^{*}$ \\
\hline \multicolumn{11}{|l|}{ 3. JUSTIFICATON OF WIFE BEATING(MALE) } \\
\hline \multicolumn{11}{|l|}{ NO;REF] } \\
\hline YES & $-0.28(0.11\}$ & $0.76(0.61 \cdot 0.95)^{*}$ & $0.15(0.21\}$ & $1.16(0.77 \cdot 1.76)$ & $0.39(0.15)$ & $1.48(1,10-1.99\}^{*}$ & $0.18(0.11)$ & $1.20\{0.97 \cdot 1.50\}$ & $-0.45[0.17]$ & $0.64\{0.46 \cdot 0.89\}^{*}$ \\
\hline \multicolumn{11}{|l|}{ 4. SOCIETY JUSTIFICATION OF WIFE BEATING } \\
\hline \multicolumn{11}{|l|}{ NO;REF] } \\
\hline YES & $0.65(0,12\}$ & $1.92(1.53 \cdot 2.42)^{*}$ & $0.85[0.17\}$ & $2.33(1,67 \cdot 3.26)^{*}$ & $0.17[0.17]$ & $1.18(0.85-1.64)$ & $0.32(0.12)$ & $1,37(1,09-1,73)^{*}$ & $* 0.56(0.13)$ & $1.75[1,37 \cdot 2,24\}^{*}$ \\
\hline & & & & & & & & & & \\
\hline \multicolumn{11}{|l|}{ MODERATOR EFFECT } \\
\hline RURALX ALCOHOL & $-0.16(0,27)$ & $0.85(0.50-1.45)$ & $0.12(0.33)$ & $1.12(0,59 \cdot 2,13\}$ & $-0.16[0.32$ & $\{0,86\{0,46-1.59\}$ & $0.03(0.22)$ & $1.03(0.67 \cdot 1.57\}$ & $-0.75\{0.22\}$ & $0.46(0,31-0.72)^{*}$ \\
\hline JUSTIFICATION OF WIFE BEATTNGX ALCOHOL & $-0.20\{0.26\}$ & $0.82(0,50-1,35\}$ & $0.68(0.45)$ & $1.98(0.83-4.75)$ & $0.48(0.32$ & $1.62(0,87 \cdot 3,03)$ & $.0 .43(0,24)$ & $0.65(0.41-1.04)$ & $-0.11(0.35)$ & $0.90,0.45-1.78$ \\
\hline SOCIETY JUSTIFICATION OF WIFE BEATINGX ALC & $0.82\{0.27\}$ & $2.26[1,33-3.86]^{*}$ & $-1.06[0.36)$ & $0.35[0.17 \cdot 0.70\}$ & $0.21[0.37$ & $1.24(0.60-2.53)$ & $-0.07(0.25)$ & $0.93(0.58-1.51)$ & $-0.20\{0.26\}$ & $0,82(0,49-1,36)$ \\
\hline
\end{tabular}

$* \mathrm{p}<0.05$ 


\section{Table 3b: Moderator Effects Of Society Level Factors On The Alcohol-Ipv Link In Ssa}

\begin{tabular}{|c|c|c|c|c|c|c|c|c|c|c|}
\hline \multicolumn{11}{|l|}{ BLOCK 1:MAIN EFFECTS } \\
\hline & NIGERIA & & \multicolumn{2}{|c|}{ SAO TOME AND PRINCIPE } & TANZANIA & & ZAMBAA & & ZIMBABWE & \\
\hline & $B[S E]$ & EXP B $[95 \% \mathrm{Cl}]$ & $\mathrm{B}(\mathrm{SE})$ & EXP B $[95 \% C \mathrm{C}]$ & $\mathrm{B}$ (SE) & EXP B $[95 \% \mathrm{C}]$ & $B(S E)$ & EXP B $[95 \% \mathrm{Cl}]$ & $B / S E]$ & EXP B $[95 \% \mathrm{Cl}]$ \\
\hline \multicolumn{11}{|l|}{ 1. ALCOHOL } \\
\hline \multicolumn{11}{|l|}{ NON ABUSE [REF] } \\
\hline ABUSE & $1.28(0.09)$ & $3.59(3.04-4.25)^{*}$ & $1.58(0.20)$ & $4.87(3,33 \cdot 7.14)^{*}$ & $1.02,0.15 \mid$ & $2.76[2,05-3.72)^{*}$ & $0.63(0,08)$ & $1.87[1.59-2.21)^{*}$ & $* 0.68(0,09)$ & $1.98(1,67 \cdot 2,35)^{*}$ \\
\hline \multicolumn{11}{|l|}{ 2. PLACE OF RESIDENCE } \\
\hline \multicolumn{11}{|l|}{ URBAN, REF] } \\
\hline RURAL & $0.21[0.07)$ & $1.23,1,08-1,41)^{*}$ & $-0.65(0.19)$ & $0.52(0.36 \cdot 0.75)^{*}$ & $-0.01[0.17]$ & $0.99(0.70-1.37)$ & $-0,44[0,09)$ & $0.64(0.54 \cdot 0.77)^{*}$ & $* \mid 0.28(0,10]$ & $1.32(1.09-1.60)$ \\
\hline \multicolumn{11}{|l|}{ 3. JUSTIFICATION OF WIFE BEATINGG(MALE) } \\
\hline \multicolumn{11}{|l|}{ NO/REF] } \\
\hline YES & $-0.11[0.08\}$ & $0,90 \mid 0.77-1.05$ & $-0.46(0.29)$ & $0.63|0.36-1.12|$ & $0.01[0.17]$ & $1.01(0.73-1.42)$ & 0.2010 .101 & $1.22\{1,01-1,48\}^{*}$ & $* \cdot-0.22,0.17]$ & $0.80\{0.64-1.01\}$ \\
\hline \multicolumn{11}{|l|}{ 4. SOCIETY JUSTIFICATION OF WIFE BEATING } \\
\hline \multicolumn{11}{|l|}{ NO/REF] } \\
\hline YES & $0,43,0.07]$ & $1.53(1,33-1,7]^{*}$ & $0.87 \mid 0.24)$ & $2,38(1,49-3,79)^{*}$ & $0.69(0.18)$ & $1.99[1.39-2.85]^{*}$ & $0.38(0,12)$ & $1,47[1,17 \cdot 1,84)^{*}$ & $* 0.71[0,11\}$ & $2,03,1,65 \cdot 2.50)^{*}$ \\
\hline \multicolumn{11}{|l|}{ MODERATOR EFFECT } \\
\hline RURALX ALCOHOL & $0.67 \mid 0.19)$ & $1.96[1.36-2.82]^{*}$ & $-0.77(0.39)$ & $0.46(0,22-0.99)^{*}$ & $0.52(0.37)$ & $1.67 \mid 0.81 \cdot 3.42]$ & $-0.37(0.18$ & $0.69(0.48-0.99)^{*}$ & $* \cdot-0.14(0,19)$ & $0.87 \mid 0.60-1.28)$ \\
\hline JUSTIFICATION OF WIFE BEATINGX ALCOHOL & $-0.20 ; 0.22\}$ & $0.82(0.53-1.27)$ & $0.49(0,60)$ & $1.62(0.50 .5 .29)$ & $0.09(0.37)$ & $1.10 \mid 0.54-2.25)$ & $0.26(0.20)$ & $1.29(0.88-1.90)$ & $-0.09 \mid 0.23\}$ & $0.91|0.58-1.43|$ \\
\hline SOCIETY JUSTIFICATION OF WIFE BEATINGX ALCOHOL & $-0.19(0.22)$ & $0.83(0.54-1.28)$ & $0.97 \mid 0.52\}$ & $0.38(0.14-1.05)$ & $-0.38(0.39)$ & $0.69(0,32-1,48)$ & $-0.46(0.23)$ & $0.63(0.40-0.99) *$ & $*|-0.29| 0.21\}$ & $0.75|0.99-1.14|$ \\
\hline
\end{tabular}


Table 3c: Model Summary

\begin{tabular}{|c|c|c|c|c|}
\hline \multicolumn{5}{|l|}{ MODEL SUMMARY } \\
\hline TIVUEL SOVIVIFITI & Chi Square & Cox\&Snell R2 & Nigelkerke R2 & \% Cases correctly classified \\
\hline BURKINA FASO & $X 2(7, N=3723)=203.20, p=0.000$ & 0.057 & 0.095 & $83.40 \%$ \\
\hline GHANA & $X 2(7, N=1019)=88.72, p=0.000$ & 0.096 & 0.131 & $66.60 \%$ \\
\hline KENYA & $X 2(7, N=1250)=50.93, p=0.000$ & 0.045 & 0.06 & $62.60 \%$ \\
\hline LIBERIA & $X 2(7, N=2197)=65.89, p=0.000$ & 0.035 & 0.046 & $57.90 \%$ \\
\hline MALAWI & $X 2(7, N=3353)=111.07, p=0.000$ & 0.036 & 0.049 & $65.60 \%$ \\
\hline NIGERIA & $X 2(7, N=6529)=283.68, p=0.000$ & 0.05 & 0.072 & $72.70 \%$ \\
\hline SAO TOME\& PRINCIPE & $X 2(7, N=821)=105.70, p=0,000$ & 0.144 & 0.199 & $71.20 \%$ \\
\hline TANZANIA & $X 2(7, N=978)=70.45, n=0.000$ & 0.078 & 0.104 & $63.60 \%$ \\
\hline ZAMBIA & $X 2(7, N=2681)=125.11, p=0.000$ & 0.051 & 0.068 & $58.60 \%$ \\
\hline ZIMBABWE & $X 2(7, N=2477)=137,9, p=0.000$ & 0.059 & 0.079 & $60.80 \%$ \\
\hline
\end{tabular}

\section{DISCUSSION}

\section{Moderators of the Alcohol-Intimate Partner Violence Link in Sub-Saharan Africa}

In this study, nine variables were studied for possible moderation of the alcohol-intimate partner violence link in sub-Saharan Africa, with each of these variables classified using the socio-ecological framework as individual level, relationship level and societal level moderators. Six of these variables showed moderator effects in some of the countries in the study, with some of these variables showing different moderator effects in different countries.

\section{Individual Level Moderators}

In Burkina Faso, Kenya, Tanzania, and Sao Tome and Principe, wealth index was found to reduce the strength of the alcohol-intimate partner violence link, with husbands/partners who are in the rich wealth index group less likely to perpetrate alcohol-related violence. This is in agreement with the report of Foran et al. (2012), who discovered that the alcohol-intimate partner violence link was weakened by higher family income. This could be because husband's drinking may lead to arguments about money in poor households which can lead to IPV, while husband's drinking in the rich wealth index may not often result in arguments since in spite of his drinking, funds are available to take care of family needs. In contrast, being in the rich index group increased the strength of the alcohol-intimate partner violence link in Liberia. This different moderation effect observed for Liberia can be explained by the political and economic situations in the country. Liberia is a country that has been in civil war for several years, leading to extreme poverty in the country. This can be seen in the gross national income per capita of $\$ 540$ (WHO, 2013). It is possible that alcohol-related violence is stronger in the rich index group because since the majority of the population are living in poverty, people who drink are 
those with high income; hence, drinking may be associated with affluence in Liberia. The absence of moderation effects of the wealth index in the other five countries is consistent with that reported by Tumwesigye et al. (2012), who on their analysis of the demographic and health survey of Uganda, reported that wealth index did not significantly moderate the association between alcohol and intimate partner violence.

In contrast with the report of Cunradi et al. (2009), employment status did not statistically moderate the alcohol-intimate partner violence link in any of the ten countries included in this study. Similar to employment status, occupation did not emerge as a significant moderator of the alcohol-IPV link in any of the countries included in this study, implying that occupation and employment status may not be as important as wealth index in determining the strength of the alcohol-IPV link in sub-Saharan Africa. It appears like whether a man is employed or not and whatever kind of job he has does not matter, but whether the family has enough income to take care of their needs is an important factor in the alcohol-IPV link in SSA.

\section{Relationship Factors}

Consistent with the findings of Foran et al. (2012), the strength of the alcohol-IPV link was weakened by increased marital length in Burkina Faso. It can be argued that couples who have stayed longer in marriage will understand each other better, thereby reducing marital conflict. It is also possible that older men may be more likely to control their behaviors when they are drunk, thereby reducing the risk of intimate partner violence. In contrast, the strength of the alcohol-IPV link was increased by longer marital length in Nigeria and Zambia.

While jealousy did not emerge as a significant moderator of the alcohol-intimate partner violence link in nine countries, it increased the strength of the association between alcohol and intimate partner violence in Tanzania. This suggests that men who abuse alcohol and exhibit jealousy are at an increased risk of perpetrating intimate partner violence than men without jealousy. This is consistent with the work of Foran and O'Leary (2008), who reported that men with high levels of jealousy were at an increased risk of perpetrating severe alcohol-related violence. The non-significant moderator effect observed in the other nine countries can be explained by the threshold conceptualization (Fals-Stewart, 2005). It could be that because the relationship between alcohol and intimate partner violence is already very strong in these sites, the presence of jealousy does not contribute any significant additional risk to this relationship.

In nine out of the ten countries included in this study, marital status did not show statistical moderation of the alcohol-intimate partner violence link. This is consistent with the results of the meta-analysis conducted by Foran and O'Leary (2008), which found no significant difference in the risk of alcohol-related intimate partner violence between married and cohabiting couples. In contrast, cohabiting couples were found to be at a reduced risk of alcohol-related intimate partner violence in Kenya. Divorce is usually frowned upon in African societies and because of this, married women often feel obliged to stay in abusive relationships. It is possible that because people who are cohabiting are not bound by societal notions about marriage, it is easier for them to leave an abusive relationship, hence the observed reduction in the risk of alcohol-related IPV.

\section{Societal Level Moderators}

In Nigeria, living in the rural area increased the strength of the alcohol-intimate partner violence link. This may be as a result of the fact that poverty abounds in the rural areas of 
Nigeria with the majority of the men unemployed or employed in a manual occupation. This has been reported to increase the risk of IPV. This agrees with the McKinney et al. (2012) findings, which reported that the association between alcohol and intimate partner violence increases with neighborhood poverty. Alcohol is also readily available tapped as palm wine, affordable and part of everyday life in rural communities in Nigeria, compared to urban areas. This increase in the availability of alcohol in the rural areas increases the likelihood of alcoholrelated violence. This explanation is consistent with the argument put forward by Cunradi (2012) and is found in general aggression literature that alcohol-related violence increases with the availability of alcohol in a neighborhood. In contrast, living in a rural area weakened the alcohol-IPV link in Malawi, Sao Tome and Principe, and Zambia. This negative moderation effect of rural dwelling in these countries can be explained by the social disorganization theory (Cunradi, 2010). Compared to the rural areas in these countries, the urban centers comprise ghettos and shanty towns, while the rural dwellers live in more organized neighborhoods and are hence more likely to be concerned about the social implications of alcohol abuse and intimate partner violence.

Male justification of the use of violence did not emerge as a significant moderator of the alcohol-IPV link in any of the ten countries included in this study. This is in contrast with results presented in previous studies. Specifically, Levinson et al. (2011) and Moore et al. (2011) reported that alcohol increased violence in men who approved of the use of violence than those without such beliefs. The observed differences in these results can be attributed to the methodologies used in these studies. While this study used data from large cross-sectional studies using a probability sample, the Levinson et al. study (2011) was experimental and used a convenient sample of 21 to 35-year-old men. On the other hand, the Moore et al. study used a small sample size comprising only college students. Because of the homogenous sample and the small sample size used in these two studies, the results may not be generalizable to other populations.

While society's justification of wife beating increased the odds of alcohol-related intimate partner violence in Burkina Faso, surprisingly, it increased the strength of the alcohol-IPV link in Zambia and Ghana. This is in agreement with the explanations found in existing literature that while justification of the use of violence can increase the odds of alcohol-related violence in some cultures, it can act as a protective factor in others because of a greater punishment being meted out to the offender (WHO, 2010; Leonard, 2005).

\section{CONCLUSION}

The purpose of this research work was to identify the possible moderators between alcohol and intimate partner violence in sub-Saharan Africa. The results of this study show that being rich decreases the odds of alcohol-related IPV in Burkina Faso. Of the three individual factors investigated for moderator effect, only wealth index moderated the alcohol-IPV link in Kenya: $\mathrm{OR}=0.53$ (95\% CI, 0.28-0.99), Tanzania: (OR=0.41, 95\% CI, 0.20-0.84) and Liberia: $(\mathrm{OR}=2.04,95 \% \mathrm{CI}, 1.16-3.59)$, with moderation reaching statistical significance in the rich index group. This result shows that being rich reduces the odds of alcohol-related violence in Kenya and Tanzania while it increases the likelihood of alcohol-related IPV in Liberia. Occupation and employment status did not moderate the alcohol-IPV link in any of the ten 
countries in this study. In Nigeria, living in rural areas increased the strength of the alcoholintimate partner violence link.

The findings of this study have the potential importance of guiding interventional programmes aimed at tackling alcohol-related IPV in multifaceted levels.

\section{REFERENCES}

Bangdiwala, S.I., Taylor, B.B. and Shankar, V. (2011) Alcohol drinking behaviour patterns and intimate partner violence. Injury Prevention, 16(Supplement 1), pp. A187-A187

Cunradi, C.B. (2009) Intimate partner violence among Hispanic men and women: the role of drinking, neighborhood disorder, and acculturation-related factors. Violence and victims, 24(1), pp. 83-97

Cunradi, C.B. (2010) Neighbourhoods, alcohol outlets and intimate partner violence: addressing research gaps in explanatory mechanisms. International journal of environmental research and public health, 7(3), pp. 799-813.

Cunradi, C.B., Mair, C., Ponicki, W. and Remer, L. (2012) Alcohol outlet density and intimate partner violence-related emergency department visits. Alcoholism, Clinical and Experimental Research, 36(5), pp. 847-853

Demographic and Health Survey (2012). Questionnaires. http://www.measuredhs.compubs/pdf/DHSQ6/DHS6_Questionnaires_5Nov2012DHSQ 6.pdf(Accessed 20/5/2013)

Fals-Stewart, W., Leonard, K. and Birchler, G. (2005). The occurrence of male-to-female intimate partner violence on days of men's drinking: The moderating effects of antisocial personality disorder. Journal of consulting and clinical psychology, 73(2), pp. $239-248$

Foran, H.M. and O'Leary, K.D. (2008) Alcohol and intimate partner violence: A metaanalytic review. Clinical psychology review, 28(7), pp. 1222-1234

Foran, H.M., Heyman, R.E., Smith Slep, and A.M., Snarr, J.D. and United States Air Force Family Advocacy Research Program (2012) Hazardous alcohol use and intimate partner violence in the military: understanding protective factors. Psychology of addictive behaviors : Journal of the Society of Psychologists in Addictive Behaviors, 26(3), pp. 471

Gage, A.J. (2005) Women's experience of intimate partner violence in Haiti.Social science \& medicine, 61(2), pp. 343-364.

Kishor, S. and Johnson, K. (2004). Risk factors for the experience of domestic violence.Profiling domestic violence: a multi-country study.Calverton, Maryland: Measure DHS ORC Macro pp. 27-52.

Krug, E.G., Mercy, J.A., Dahlberg, L.L. and Zwi, A.B. (2002). The world report on violence and health.The lancet, 360(9339), pp. 1083-1088 .

Leonard, K.E. (2005) Alcohol and intimate partner violence: when can we say that heavy drinking is a contributing cause of violence? Addiction (Abingdon, England) [online], 100(4), pp. 422-425

Levinson, C.A., Giancola, P.R. and Parrott, D.J. (2011) Beliefs about aggression moderate alcohol's effects on aggression. Experimental and clinical psychopharmacology [online], 19(1), pp. 64-74 
Moore, T., Elkins, S., McNulty, J., Kivisto, A. and Handsel, V. (2011) Alcohol Use and Intimate Partner Violence Perpetration Among College Students: Assessing the Temporal Association Using Electronic Diary Technology. PSYCHOLOGY OF VIOLENCE, 1(4), pp. 315-328

Stith, S.M., Smith, D.B., Penn, C.E., Ward, D.B. and Tritt, D. (2004) Intimate partner physical abuse perpetration and victimization risk factors: A meta-analytic review. Aggression and Violent Behavior, 10(1), pp. 65-98

Taft, C.T., O'Farrell, T.J., Doron-LaMarca, S., Panuzio, J., Suvak, M.K., Gagnon, D.R. and Murphy, C.M. (2010) Longitudinal risk factors for intimate partner violence among men in treatment for alcohol use disorders. Journal of consulting and clinical psychology [online], 78(6), pp. 924-935

Trocki, K.F. and Caetano, R. (2003) Exposure to family violence and temperament factors as predictors of adult psychopathology and substance use outcomes. Journal of Addictions Nursing, 14(4), pp. 183-192

Tumwesigye, N.M., Kyomuhendo, G.B., Greenfield, T.K. and Wanyenze, R.K. (2012) Problem drinking and physical intimate partner violence against women: evidence from a national survey in Uganda. BMC public health, 12(1), pp. 399-399.

Uthman, O.A., Lawoko, S. and Moradi, T. (2010) Sex disparities in attitudes towards intimate partner violence against women in sub-Saharan Africa: a socio-ecological analysis.BMC Public Health, 10(1), pp. 223-223

Welman, C., Huysamen, G.K., Kruger, F. and Mitchell, B. (2005) Research methodology. Oxford: Oxford University Press

World Health Organisation (2013), Global Health Observatory. http://apps.who.int/gho/data/node.country_(Accessed 10/10/2013)

World Health Organization (2010). Preventing intimate partner and sexual violence against women: Taking action and generating evidence. Injury Prevention, 16, pp359-360

World Health Organization (2012). Intimate Partner Violence and Alcohol Fact sheet http://www.who.int/violence_injury_prevention/violence/world_report/factsheets/ft_inti mate.pdf (Accessed 20/5/2013) 